\title{
Index of convexity: A novel method for assessing liver functional reserve using technetium-99m-galactosyl human serum albumin liver scintigraphy
}

\author{
Takafumi Sato $^{1}$, Junichi Arita ${ }^{2}$, Yosuke Inoue ${ }^{1}$, Rintaro Koga ${ }^{1}$, Yu Takahashi ${ }^{1}$, Akio Saiura $^{1, *}$ \\ ${ }^{1}$ Department of Gastroenterological Surgery, Cancer Institute Hospital of the Japanese Foundation for Cancer Research, Tokyo, \\ Japan; \\ ${ }^{2}$ Hepato-Biliary-Pancreatic Division, Department of Surgery, The University of Tokyo, Tokyo, Japan.
}

\begin{abstract}
Summary Preoperative evaluation of liver functional reserve is important in hepatobiliary surgery. Although the indocyanine green retention rate at 15 minutes (ICG-R15) is the gold standard for this purpose, a new method without technical complexity would be preferable. We assessed the usefulness of the previously established index of convexity (IOC). In total, 159 consecutive patients who underwent both technetium-99m-galactosyl human serum albumin ${ }^{99 \mathrm{~m}}$ Tc-GSA) scintigraphy and the ICG-R15 were included. Correlation coefficients between indices from ${ }^{99 \mathrm{~m}}$ Tc-GSA scintigraphy and blood examinations including ICG-R15 were evaluated, and a conversion formula from the IOC to the ICG-R15 was established. The IOC was calculated as $[L(15) \times 2-L(3)-L(27)] /[L(27)-L(3)]$, where $L(t)$ indicates the radiation counts within the whole liver at $t$ minutes after ${ }^{99 m}$ Tc-GSA injection. The IOC showed a significantly stronger correlation with the ICG-R15 $(r=-0.532, p<0.001)$ than the index of blood clearance (HH15) and the receptor index (LHL15). A formula for estimating ICG-R15 from IOC was "ICG-R15 = -31.0 $\times$ IOC +30.1 ". In conclusion, the IOC is a better index for evaluating preoperative liver functional reserve than the conventional indices. A formula for estimating ICG-R15 from the IOC will be useful.
\end{abstract}

Keywords: Preoperative assessment, liver function, hepatectomy, GSA scintigraphy

\section{Introduction}

Preoperative evaluation of liver functional reserve is important to predict severe complication after hepatectomy, which has been more enhanced because the indication for hepatic resection has been expanded along with the development of surgical techniques and perioperative management protocols (1-5). Among many indicators of liver functional reserve $(3,4,6-13)$, the indocyanine green retention rate at 15 minutes (ICG-R15) is the gold standard technique $(1,14,15)$. However, the patients should be rested for 2 to 3 hours in a horizontal

Released online in J-STAGE as advance publication May 8, 2017.

*Address correspondence to:

Dr. Akio Saiura, Department of Gastroenterological Surgery, Cancer Institute Hospital of the Japanese Foundation for Cancer Research, 3-8-31 Ariake, Koto-ku, Tokyo 135-8550, Japan.

E-mail: akio.saiura@jfcr.or.jp position and the pretest fasting is necessary before ICG-R15 test, which usually necessitates hospitalization of the patients. Moreover, the technique of ICG-R15 test is somewhat complex because three times of blood sampling after injecting ICG should be performed accurately with time-lag less than a few seconds. Additionally, the results can be inconclusive in patients with obstructive jaundice or congenital ICG excretory defects (16). Another auxiliary or even alternative examination for estimating the liver functional reserve is desired.

Technetium-99m diethylenetriamine-penta-acetic acid-galactosyl human serum albumin ( $\left.{ }^{99 \mathrm{~m}} \mathrm{Tc}-\mathrm{GSA}\right)$ scintigraphy is one of the prevalent examinations performed for evaluation of liver functional reserve (1725). Conventional indices of ${ }^{99 \mathrm{~m}} \mathrm{Tc}-\mathrm{GSA}$ scintigraphy, namely the blood clearance index (HH15) and the receptor index (LHL15), use accumulation counts of only two time points. The index of convexity (IOC) was proposed by Miki et al. (26) as a novel alternative to 
HH15 and LHL15. The IOC is calculated from hepatic accumulation counts on GSA scintigraphy at three fixed time points, surrogating the convexity of the hepatic accumulation curve of GSA scintigraphy. This index was created based on data obtained in patients, most of whom (68.5\%) had hepatocellular carcinoma. However, more and more patients with liver metastases from colorectal carcinoma undergone hepatic resection thanks to advent of preoperative chemotherapy. In this study, we assessed the usefulness of the IOC utilizing a patient cohort including many patients with colorectal liver metastases. In addition, we propose a new conversion formula from the IOC to the ICG-R15.

\section{Materials and Methods}

\subsection{Patients and data collection}

A total of 159 consecutive patients underwent both ${ }^{99 \mathrm{~m}} \mathrm{Tc}-$ GSA scintigraphy and ICG-R15 test within one month before hepatic resection from June 2011 to October 2012 at the Cancer Institute Hospital of the Japanese Foundation for Cancer Research. Two patients who were diagnosed with constitutional ICG excretory defect were excluded from the study. The ${ }^{99 \mathrm{~m}} \mathrm{Tc}-\mathrm{GSA}$ scintigraphy was incorporated into the routine preoperative examination in our hospital from 2011 after private communication with the creator of this index; the index was published in the literature in 2013 (26). The IOC, ICG-R15, and other clinical data were collected from a retrospective review of the clinical records. The indices calculated from ${ }^{99 \mathrm{~m}} \mathrm{Tc}-\mathrm{GSA}$ scintigraphy were HH15, LHL15, and IOC. The serum levels of total bilirubin (T-Bil), direct bilirubin, aspartate aminotransferase (AST), alanine aminotransferase (ALT), choline esterase, albumin (Alb), transthyretin (TTR), total cholesterol (T-Cho), prothrombin time-international normalized ratio (PT-INR), and platelet count (Plt) were determined after admission. Histopathological examination of the noncancerous part of the resected liver was performed to estimate liver impairment.

\section{2. ${ }^{99 m} T c-G S A$ scintigraphy}

After intravenous injection of $3 \mathrm{mg}$ of ${ }^{99 \mathrm{~m}} \mathrm{Tc}-\mathrm{GSA}$ ( $1 \mathrm{ml}$, 185 MBq; Nihon Medi-Physics Co., Ltd., Nishinomiya, Japan), dynamic images were recorded with the patient in the supine position using a gamma camera (Infinia 3; GE Healthcare, Chicago, IL, USA) under a large field of view. Digital images were acquired at the rate of 30 seconds per frame until 30 minutes after the injection. The regions of interests (ROIs) were determined by specialized medical engineers. The radiation counts obtained from the ROIs for the whole liver and heart were recorded at each time point. The data were processed on a workstation (GENIE Xeleris, version 3.1; GE Healthcare).

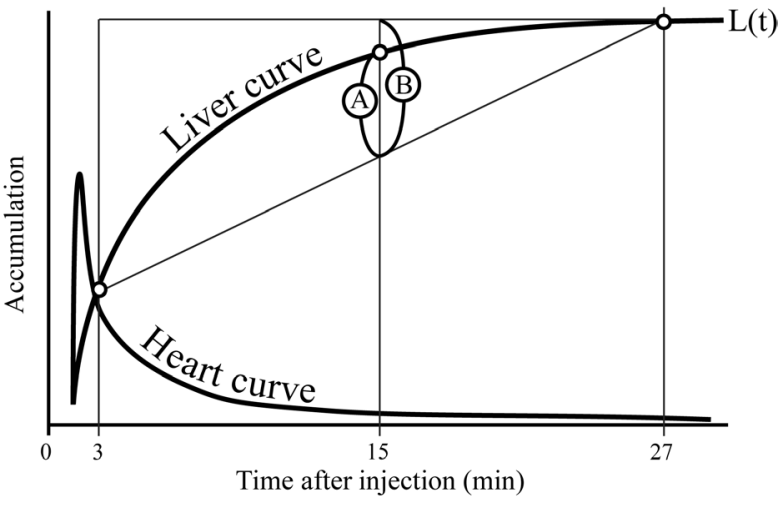

Figure 1. Representative accumulation curves and time points for calculation of the index of convexity. The solid line is the liver accumulation curve, and the dashed line is the heart curve. The index of convexity is the ratio of line $\mathbf{A}$ to $\mathbf{B}$. A higher value indicates better liver function.

\subsection{IOC}

The IOC was calculated as $[\mathrm{L}(15) \times 2-\mathrm{L}(3)-\mathrm{L}(27)]$ / [(L(27) - L(3)], the HH15 was calculated as H(15) / $\mathrm{H}(3)$, and the LHL15 was calculated as L(15) / [L(15) $+\mathrm{H}(15)]$, where $\mathrm{L}(\mathrm{t})$ and $\mathrm{H}(\mathrm{t})$ indicate the radiation counts at $\mathrm{t}$ minutes after ${ }^{99 \mathrm{~m}} \mathrm{Tc}-\mathrm{GSA}$ injection within the whole liver and whole heart, respectively. This index is a surrogate for the numerical index of the convexity of the curve of liver radiation counts until 27 minutes after injection (26). The IOC was devised using line ratio: the ratio of each distance between the midpoint of $\mathrm{L}(3)$ and $\mathrm{L}(27)$ to $\mathrm{L}(15)$ (Figure 1A) and the midpoint of L(3) and $\mathrm{L}(27)$ to $\mathrm{L}(27)$ (Figure $1 \mathrm{~B})$.

\subsection{Statistical analysis}

The correlation between the indices from ${ }^{99 \mathrm{~m}} \mathrm{Tc}-\mathrm{GSA}$ scintigraphy and all other indices were analyzed using Spearman's rank correlation coefficient test as a nonparametric test. A linear model was calculated by simple and multiple regression analyses with a stepwise method using significant $p$ values. Subanalyses comparing liver functional indices were performed after the division of the patients into the following groups: first and repeat hepatectomy groups, non-chemotherapy and chemotherapy groups, and non-cirrhosis and cirrhosis groups. The correlations among the indices were also analyzed using Spearman's rank correlation coefficient test. All statistical analyses were performed using IBM SPSS Statistics for Windows, version 23.0 (IBM Corp., Armonk, NY, USA). Statistical significance was established at $p<0.05$

\section{Results}

\subsection{Patient characteristics}

Table 1 shows the background of the patients. 
Table 1. Summary of patient characteristics

\begin{tabular}{lc}
\hline Age (Median, range) & $64(29-84)$ \\
Sex (Male/Female) & $116 / 43$ \\
Preoperative Chemotherapy (+/-) & $51 / 108$ \\
Duration between ICG and GSA scintigraphy (days, mean \pm SD) & $10.1 \pm 8.2$ \\
Times of hepatectomy (First/Repeat) & $129 / 30$ \\
Diagnosis $(n, \%)$ & \\
Metastatic liver tumor & $87(54.7 \%)$ \\
$\quad$ Colorectal cancer & $73(83.9 \%)$ \\
Gastric cancer & $5(5.7 \%)$ \\
Lung cancer & $3(3.4 \%)$ \\
Renal cell cancer & $2(2.3 \%)$ \\
Breast cancer & $1(1.1 \%)$ \\
Lingual cancer & $1(1.1 \%)$ \\
$\quad$ Pancreatic neuroendocrine tumor & $1(1.1 \%)$ \\
Duodenal carcinoid & $1(1.1 \%)$ \\
Hepatocellular carcinoma & $53(33.3 \%)$ \\
Cholangiocarcinoma & $17(10.7 \%)$ \\
Others & $2(1.3 \%)$ \\
Histopathological findings of the liver $(n, \%)$ & $119(74.8 \%)$ \\
Normal liver & $4(2.5 \%)$ \\
Fatty liver & $10(6.3 \%)$ \\
Chronic hepatitis & $26(16.4 \%)$ \\
Liver cirrhosis & \\
\hline
\end{tabular}

SD: standard deviation, ICG: indocyanine green clearance test, GSA scintigraphy: technetium-99m-galactosyl human serum albumin liver scintigraphy.

Preoperative chemotherapy was performed in $32 \%$ and a repeat hepatectomy (i.e., second time or more) was performed in $19 \%$ of all patients. $55 \%$ of all patients had liver metastasis, 33\% had hepatocellular carcinoma, and $11 \%$ had cholangiocarcinoma. Among 87 patients with liver metastasis, colorectal carcinoma was the origin of the liver metastasis in 73 patients $(84 \%)$. Of these 73 patients, preoperative chemotherapy was administered to 51 patients: 5-fluorouracil, leucovorin, plus oxaliplatin (FOLFOX) in 23 patients, capecitabine plus oxaliplatin (CapeOX) in 11, capecitabine in 6 , 5-fluorouracil, leucovorin, plus irinotecan (FOLFIRI) in four, and other regimens in seven. Molecular target drugs were used in 31 patients: anti-vascular endothelial growth factor antibody (bevacizumab) in 18 and antiepidermal growth factor receptor antibody (cetuximab or panitumumab) in 11.

Postoperative histopathological examination of the noncancerous part of the resected liver revealed normal liver in 119 patients, fatty liver in 4 , chronic hepatitis in 10 , and liver cirrhosis in 26 . The cirrhosis group included patients with liver cirrhosis and chronic viral hepatitis, and the non-cirrhosis group included the remaining patients with normal and fatty liver (Table 1).

\subsection{Correlation between the GSA scintigraphy indices and other indices}

Scatter diagrams of the ICG-R15 and each index obtained from ${ }^{99 \mathrm{~m}} \mathrm{Tc}-\mathrm{GSA}$ scintigraphy, namely, IOC, HH15, and LHL15, in all patients group are shown in Figure 2. Scatter diagrams of subgroups are shown in Figure 3. Table 2 shows the correlation coefficients between the indices obtained from ${ }^{99 \mathrm{~m}} \mathrm{Tc}-\mathrm{GSA}$ scintigraphy and other indices. The IOC showed the strongest correlation $(r=-0.532, p<0.01)$ to ICG-R15 compared with HH15 $(\mathrm{r}=0.336, \mathrm{p}<0.01)$ and LHL15 $(r=-0.348, p<0.01)$. This trend was seen in all subgroups except the cirrhosis group. The IOC showed the strongest correlation to most of other indices (total bilirubin, direct bilirubin, choline esterase, albumin, transthyretin, and Plt). Non-chemotherapy group and cirrhosis group showed stronger correlation coefficients between IOC and ICG-R15 $(r=-0.605, p<0.001 ; r$ $=-0.600, p<0.001)$. In the chemotherapy group and repeat hepatectomy group, only the IOC showed a significant correlation with the ICG-R15. ICG-R15 and Plt showed stronger correlation coefficients $(r=0.532$, $p<0.01 ; r=0.496, p<0.01)$ to the IOC followed by TTR $(r=0.420, p<0.01)$.

\subsection{Linear regression equation and conversion formula}

Based on a simple linear regression analysis, a conversion formula estimating ICG-R15 value from the IOC value was generated: "ICG-R15 $=-30.4 \times \mathrm{IOC}+$ 29.8 " $(r=0.612, p<0.001)$. Among the subgroups, the first hepatectomy group showed the highest $r$ value, and the estimation formula from the first hepatectomy group was calculated as "ICG-R15 $=-31.0 \times$ IOC $+30.1 "(r$ $=0.650, p<0.001)$. A simplified diagram estimating ICG-R15 from the IOC value is shown in Figure 4.

\section{Discussion}

In this study, the IOC showed a significant correlation with the ICG-R15, which is the current gold standard test for estimating liver functional reserve. The IOC is a surrogate for the curve convexity of continual hepatic radiation counts during 30 minutes following 

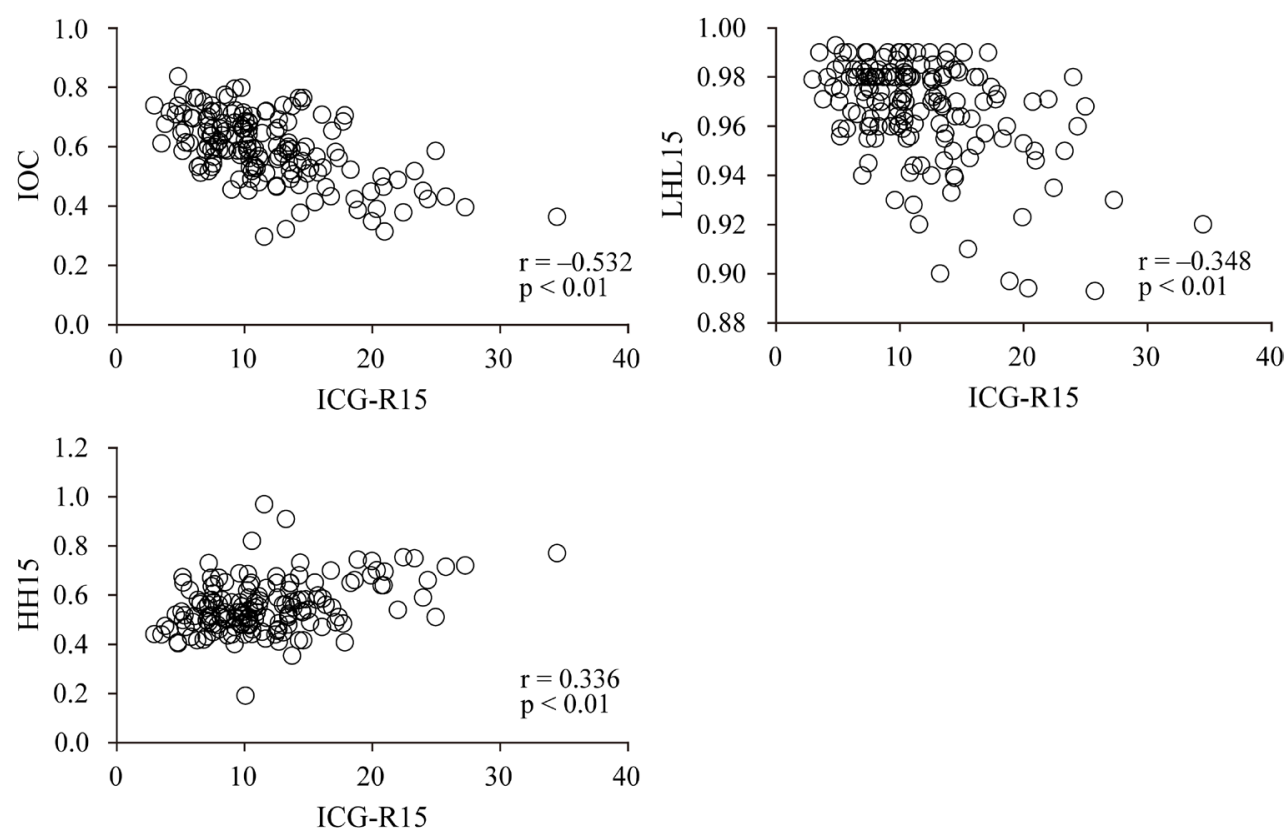

Figure 2. Scatter diagrams of the ICG-R15 and the indices of ${ }^{99 \mathrm{~m}}$ Tc-GSA scintigraphy. ICG-R15: indocyanine green retention rate at $15 \mathrm{~min}, \mathrm{GSA}$ : galactosyl human serum albumin.
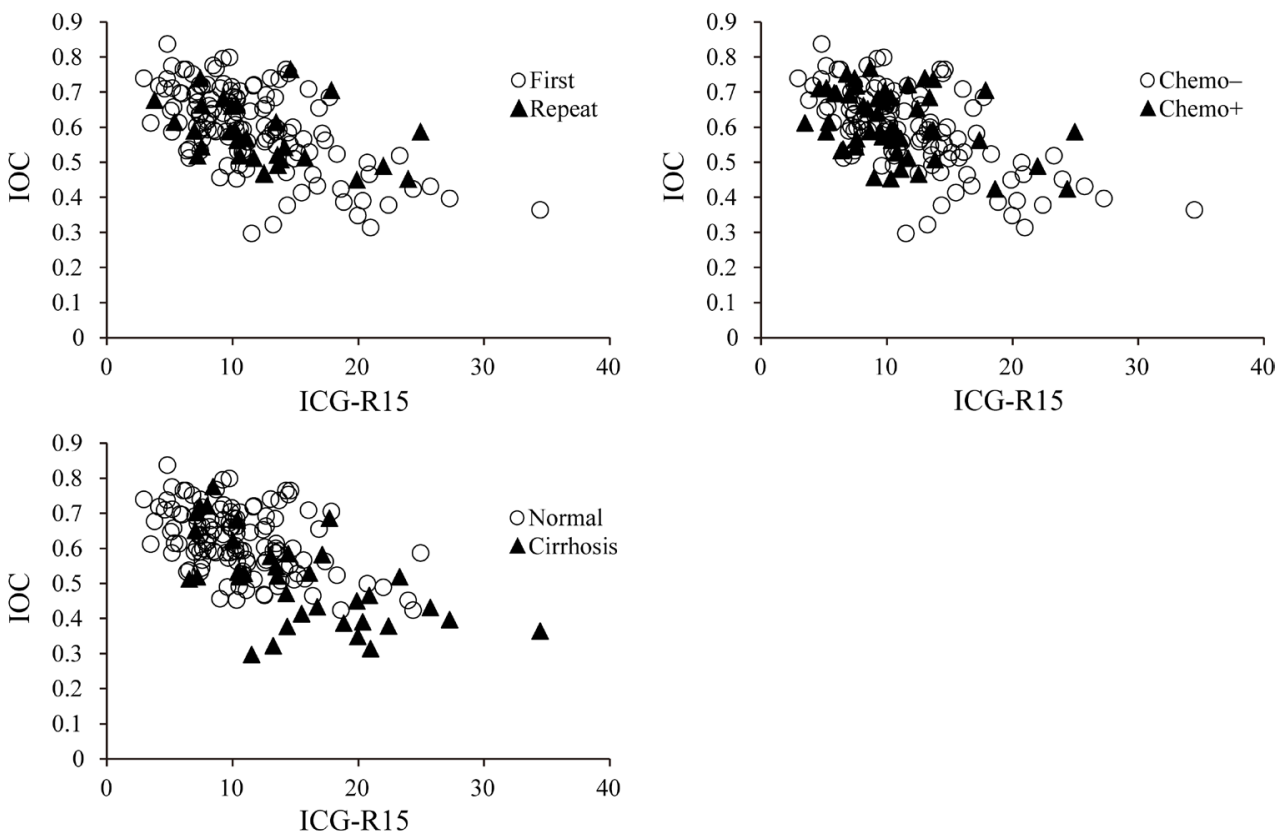

Figure 3. Scatter diagrams of the ICG-R15 and IOC for each subgroup. ICG-R15: indocyanine green retention rate at 15 min, IOC: index of convexity, First: first hepatectomy, Repeat: repeat hepatectomy, Chemo: preoperative chemotherapy, Normal: normal liver in noncancerous area of the liver, Cirrhosis: liver cirrhosis in noncancerous area of the liver.

the injection (26), of which the calculation is so complicated. This novel index uses only three hepatic radiation counts at 3,15 , and 27 minutes after injection, respectively. A larger IOC value indicates that ${ }^{99 \mathrm{~m}} \mathrm{Tc}$ GSA accumulates in the liver more rapidly and the curve of the radiation counts reaches to a plateau faster, meaning higher hepatic capacity of uptake.

The correlation coefficients between ICG-R15 and the IOC were significantly stronger than the conventional indices of HH15 and LHL15. The calculation of the IOC incorporates three radiation counts at different time points, while that of HH15 incorporates two counts. The IOC utilizes more information obtained from the scintigraphy than HH15, thus may be superior to HH15. The calculation of LHL15 incorporates a hepatic radiation count and a heart radiation count only at single time point of 15 minutes, respectively, so that IOC may be superior to LHL15 from the similar viewpoint. Furthermore, both HH15 and LHL15 use radiation counts from the heart, of which the ROI usually includes not only the heart but also the liver because the two organs are tightly adjacent. This inaccuracy of measuring 
Table 2. Correlation coefficients between the indices obtained from GSA scintigraphy and blood tests

\begin{tabular}{|c|c|c|c|c|c|c|c|c|c|c|c|c|}
\hline \multirow{2}{*}{ Items } & \multicolumn{6}{|c|}{ All Patients $(n=159)$} & \multicolumn{6}{|c|}{$\begin{array}{l}\text { Non-chemotherapy, First hepatectomy, } \\
\text { and Non-cirrhosis Group }(n=58)\end{array}$} \\
\hline & IOC & $p$ & HH15 & $p$ & LHL15 & $p$ & IOC & $p$ & HH15 & $p$ & LHL15 & $p$ \\
\hline ICG-R15 & -0.532 & $\dagger \dagger$ & 0.336 & $\dagger$ & -0.348 & $\dagger \dagger$ & -0.430 & $\dagger \dagger$ & - & n.s. & - & n.s. \\
\hline T-Bil & -0.249 & $\dagger$ & - & n.s. & - & n.s. & - & n.s. & - & n.s. & - & n.s. \\
\hline D-Bil & -0.363 & $\dagger$ & 0.271 & $\dagger$ & - & n.s. & -0.418 & $\dagger$ & - & n.s. & - & n.s. \\
\hline Cho-E & 0.292 & $\dagger$ & -0.287 & $\dagger$ & 0.185 & $*$ & - & n.s. & - & n.s. & - & n.s. \\
\hline Alb & 0.232 & $\dagger$ & -0.210 & $\dagger$ & 0.209 & $\dagger$ & - & n.s. & - & n.s. & - & n.s. \\
\hline TTR & 0.420 & $\dagger$ & -0.371 & $\dagger \dagger$ & 0.178 & $*$ & 0.391 & $\dagger$ & - & n.s. & - & n.s. \\
\hline \multirow[t]{2}{*}{ Plt } & 0.496 & $\dagger$ & -0.374 & $\dagger$ & 0.250 & $\dagger$ & 0.351 & $\dagger$ & - & n.s. & - & n.s. \\
\hline & \multicolumn{6}{|c|}{ Non-chemotherapy Group $(n=108)$} & \multicolumn{6}{|c|}{ Chemotherapy Group $(n=51)$} \\
\hline ICG-R15 & -0.605 & $\dagger \dagger$ & 0.429 & $\dagger \dagger$ & -0.392 & $\dagger \dagger$ & -0.327 & $*$ & - & n.s. & - & n.s. \\
\hline T-Bil & -0.243 & $*$ & - & n.s. & - & n.s. & - & n.s. & - & n.s. & - & n.s. \\
\hline D-Bil & -0.390 & $\dagger$ & 0.312 & $\dagger$ & - & n.s. & - & n.s. & - & n.s. & - & n.s. \\
\hline Cho-E & 0.339 & $\dagger$ & -0.365 & $\dagger$ & 0.255 & $*$ & - & n.s. & - & n.s. & - & n.s. \\
\hline Alb & 0.245 & $*$ & -0.230 & $*$ & 0.250 & $\dagger$ & - & n.s. & - & n.s. & - & n.s. \\
\hline TTR & 0.472 & $\dagger$ & -0.428 & $\dagger \dagger$ & 0.270 & $\dagger$ & - & n.s. & - & n.s. & - & n.s. \\
\hline Plt & 0.507 & $\dagger \dagger$ & -0.424 & $\dagger$ & 0.294 & $\dagger$ & 0.448 & $\dagger$ & - & n.s. & - & n.s. \\
\hline \multicolumn{7}{|c|}{ First Hepatectomy Group $(n=129)$} & \multicolumn{6}{|c|}{ Repeat Hepatectomy Group $(n=30)$} \\
\hline ICG-R15 & -0.544 & $\dagger$ & 0.352 & $\dagger \dagger$ & -0.375 & $\dagger$ & -0.477 & $\dagger$ & - & n.s. & - & n.s. \\
\hline T-Bil & -0.244 & $\dagger$ & - & n.s. & - & n.s. & - & n.s. & - & n.s. & - & n.s. \\
\hline D-Bil & -0.372 & $\dagger$ & 0.255 & $\dagger$ & - & n.s. & -0.388 & $*$ & - & n.s. & - & n.s. \\
\hline Cho-E & 0.343 & $\dagger \dagger$ & -0.350 & $\dagger$ & 0.218 & $*$ & - & n.s. & - & n.s. & - & n.s. \\
\hline Alb & 0.265 & $\dagger$ & -0.273 & $\dagger$ & 0.216 & $*$ & - & n.s. & - & n.s. & - & n.s. \\
\hline TTR & 0.440 & $\dagger$ & -0.428 & $\dagger$ & 0.268 & $\dagger$ & - & n.s. & - & n.s. & - & n.s. \\
\hline Plt & 0.492 & $\dagger$ & -0.370 & $\dagger$ & 0.242 & $\dagger$ & 0.390 & $*$ & - & n.s. & - & n.s. \\
\hline \multicolumn{7}{|c|}{ Non-cirrhosis group $(n=123)$} & \multicolumn{6}{|c|}{ Cirrhosis Group $(n=36)$} \\
\hline ICG-R15 & -0.390 & $\dagger$ & - & n.s. & -0.178 & $*$ & -0.600 & $\dagger$ & 0.440 & $\dagger$ & -0.630 & $\dagger$ \\
\hline T-Bil & -0.234 & $\dagger$ & - & n.s. & - & n.s. & - & n.s. & - & n.s. & - & n.s. \\
\hline D-Bil & -0.291 & $\dagger$ & - & n.s. & - & n.s. & - & n.s. & - & n.s. & - & n.s. \\
\hline Cho-E & - & n.s. & - & n.s. & - & n.s. & 0.360 & $*$ & -0.390 & $*$ & 0.471 & $\dagger$ \\
\hline Alb & 0.191 & $*$ & - & n.s. & - & n.s. & - & n.s. & - & n.s. & - & n.s. \\
\hline TTR & 0.339 & $\dagger$ & -0.229 & $*$ & - & n.s. & - & n.s. & - & n.s. & 0.397 & $*$ \\
\hline Plt & 0.412 & $\dagger \dagger$ & -0.268 & $\dagger$ & - & n.s. & 0.509 & $\dagger$ & -0.374 & $*$ & 0.474 & $\dagger$ \\
\hline
\end{tabular}

IOC: index of convexity, ICG-R15: indocyanine green retention rate at 15 minutes, T-Bil: total bilirubin, D-Bil: direct bilirubin, Cho-E: choline esterase, Alb: albumin, TTR: transthyretin, Plt: platelet count, ${ }^{*}: p<0.05, \uparrow: p<0.01$, ${ }^{\dagger}$ †: $p<0.001$, n.s.: not significant.

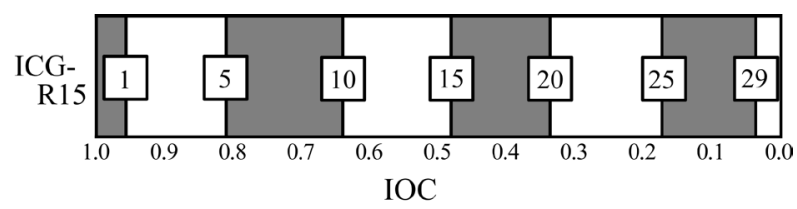

Figure 4. Simplified diagram of conversion formula from IOC to ICG-R15 in the first hepatectomy group. IOC: index of convexity, ICG-R15: indocyanine green retention rate at $15 \mathrm{~min}$.

of the radiation count may cause the difference. Another possible reason for the difference would be difference of the mechanism. The IOC focuses on the change of the rate of material uptake, while the HH15 indicates clearance rate of the material from the blood pool, and the LHL15 indicates the proportion of hepatic uptake to all injected material at one time point.

In the present study, the most common cause for hepatectomy was liver metastasis (54\%), and preoperative chemotherapy was performed in as many as $59 \%$ of these patients. The IOC was initially devised based on the data from the patients of whom $69 \%$ had hepatocellular carcinoma and thus had impaired liver (26). Nonetheless, the IOC values were well correlated with ICG-R15 values; the IOC can be applied to the patients with various kinds of disease. In our result, in spite of the small cohort with only 36 patients, the correlation coefficient between IOC and ICG-R15 in cirrhosis group was stronger than most other subgroups, which is consistent with the previous report. On the other hand, chemotherapy group showed the lowest correlation value. This trend may be because various regimens of chemotherapy were performed according to the each cause and medical history, and perhaps because small sample size. Further analysis utilizing a large number of patients undergoing chemotherapy is 
necessary.

The correlation coefficients were worse in the repeat hepatectomy group than in the first hepatectomy group. A possible cause for this is the deformity of the liver shape after the preceding hepatectomy; setting of ROI during ${ }^{99 m}$ Tc-GSA scintigraphy may be difficult in some cases. The ROI setting should be confirmed if the IOC value showed discrepancy with expected one in the patients who had undergone hepatectomy.

In the chemotherapy group, the correlation coefficients were unsatisfactory. Sinusoidal obstructive syndrome (SOS) and chemotherapy-associated steatohepatitis (CASH) are well-known side effects of chemotherapy affecting liver functional reserve (2729). SOS is characterized by obstruction of a central vein within a sinusoid, and $\mathrm{CASH}$ is caused by a metabolic disorder. The IOC indicates the rate of the material uptake and its alteration, while the ICG-R15 indicates the clearance of the material from the blood pool, deeply influenced by the velocity of portal venous flow. This difference of mechanisms may be the key for explaining this discrepancy and, perhaps, either of the two examinations may be superior to the other in patients undergoing chemotherapy. This should be further confirmed utilizing data including postoperative complication.

We created a conversion formula for estimating the ICG-R15, which so far is the gold standard for estimating liver functional reserve. Because the liver functional reserve is so complicated that no one can precisely define this term, the liver surgeons should decide surgical indication and plan for hepatic resection referring to not only ICG-R15 but also other indices. A conversion formula from the IOC value to the ICG-R15 value may be useful in comparison to ICG-R15 value. Moreover, in patients with congenital ICG excretory defect or those with portosystemic shunt, this formula may be more enhanced in estimating the liver functional reserve.

In conclusion, the IOC is superior to the conventional index obtained from ${ }^{99 \mathrm{~m}} \mathrm{Tc}-\mathrm{GSA}$ scintigraphy in terms of evaluating liver functional reserve. A conversion formula from the IOC to ICG-R15 is proposed for further utility in preoperative patient management.

\section{Acknowledgements}

We are grateful to Dr. Kenji Miki from the Department of Gastrointestinal Surgery of JR Tokyo General Hospital for his presentation of the research data and explanation of the concept and calculation method of the index of convexity before publication of his report.

\section{References}

1. Wakiya T, Kudo D, Toyoki Y, Ishido K, Kimura N, Narumi S, Kijima H, Hakamada K. Evaluation of the usefulness of the indocyanine green clearance test for chemotherapy-associated liver injury in patients with colorectal cancer liver metastasis. Ann Surg Oncol. 2014; 21:167-172.

2. Eguchi S, Takatsuki M, Soyama A, Hidaka M, Nakao K, Shirasaka T, Yamamoto M, Tachikawa N, Gatanaga H, Kugiyama Y, Yatsuhashi H, Ichida T, Kokudo N. Analysis of the hepatic functional reserve, portal hypertension, and prognosis of patients with human immunodeficiency virus/hepatitis $C$ virus coinfection through contaminated blood products in Japan. Transplant Proc. 2014; 46:736738.

3. Imura S, Shimada M, Utsunomiya T. Recent advances in estimating hepatic functional reserve in patients with chronic liver damage. Hepatol Res. 2015; 45:10-19.

4. Hoekstra LT, de Graaf W, Nibourg GA, Heger M, Bennink RJ, Stieger B, van Gulik TM. Physiological and biochemical basis of clinical liver function tests: A review. Ann Surg. 2013; 257:27-36.

5. Seyama Y, Kokudo N. Assessment of liver function for safe hepatic resection. Hepatol Res. 2009; 39:107-116.

6. Nanashima A, Abo T, Arai J, Matsumoto H, Kudo T, Nagayasu T. Functional liver reserve parameters predictive for posthepatectomy complications. J Surg Res. 2013; 185:127-135.

7. Fung J, Poon RT, Yu WC, Chan SC, Chan AC, Chok KS, Cheung TT, Seto WK, Lo CM, Lai CL, Yuen MF. Use of liver stiffness measurement for liver resection surgery: Correlation with indocyanine green clearance testing and post-operative outcome. PLoS One. 2013; 8:e72306.

8. Cao Y, Wang H, Johnson TD, Pan C, Hussain H, Balter JM, Normolle D, Ben-Josef E, Ten Haken RK, Lawrence TS, Feng M. Prediction of liver function by using magnetic resonance-based portal venous perfusion imaging. Int J Radiat Oncol Biol Phys. 2013; 85:258263.

9. Yoshida M, Shiraishi S, Sakaguchi F, Utsunomiya D, Tashiro K, Tomiguchi S, Okabe H, Beppu T, Baba H, Yamashita Y. Fused 99m-Tc-GSA SPECT/CT imaging for the preoperative evaluation of postoperative liver function: Can the liver uptake index predict postoperative hepatic functional reserve? Jpn J Radiol. 2012; 30:255262.

10. Sourbron S, Sommer WH, Reiser MF, Zech CJ. Combined quantification of liver perfusion and function with dynamic gadoxetic acid-enhanced MR imaging. Radiology. 2012; 263:874-883.

11. de Graaf W, van Lienden KP, Dinant S, Roelofs JJ, Busch OR, Gouma DJ, Bennink RJ, van Gulik TM. Assessment of future remnant liver function using hepatobiliary scintigraphy in patients undergoing major liver resection. J Gastrointest Surg. 2010; 14:369-378.

12. de Graaf W, Bennink RJ, Vetelainen R, van Gulik TM. Nuclear imaging techniques for the assessment of hepatic function in liver surgery and transplantation. J Nucl Med. 2010; 51:742-752.

13. Kubota K, Makuuchi M, Kusaka K, Kobayashi T, Miki K, Hasegawa K, Harihara Y, Takayama T. Measurement of liver volume and hepatic functional reserve as a guide to decision-making in resectional surgery for hepatic tumors. Hepatology. 1997; 26:1176-1181.

14. Derpapas MK, Contis J, Fragulidis GP, Lykoudis PM, Polymeneas G, Ntourakis S, Voros D. Correlation of the ICG test with risk factors and postoperative outcomes following hepatic resection. J BUON. 2013; 18:703-707.

15. Takamoto T, Hashimoto T, Sano K, Maruyama Y, 
Inoue K, Ogata S, Takemura T, Kokudo N, Makuuchi M. Recovery of liver function after the cessation of preoperative chemotherapy for colorectal liver metastasis. Ann Surg Oncol. 2010; 17:2747-2755.

16. Kadono J, Kumemura H, Nishida S, Nakamura N, Gejima K, Nakajo M, Tsuchimochi S, Matsumoto J, Hamada N, Sakata R. ${ }^{99 \mathrm{~m}}$ Tc-DTPA-galactosyl-humanserum-albumin liver scintigraphy for evaluating hepatic functional reserve before hepatectomy in a patient with indocyanine green excretory defect: Report of a case. Surg Today. 2006; 36:481-484.

17. Nanashima A, Tobinaga S, Abo T, Sumida Y, Araki M, Hayashi H, Sakamoto I, Kudo T, Takeshita H, Hidaka S, Sawai T, Hatano K, Nagayasu T. Relationship of hepatic functional parameters with changes of functional liver volume using technetium-99m galactosyl serum albumin scintigraphy in patients undergoing preoperative portal vein embolization: A follow-up report. J Surg Res. 2010; 164:e235-242.

18. Kaibori M, Ha-Kawa SK, Maehara M, Ishizaki M, Matsui K, Sawada S, Kwon AH. Usefulness of Tc-99mGSA scintigraphy for liver surgery. Ann Nucl Med. 2011; 25:593-602.

19. de Graaf W, Vetelainen RL, de Bruin K, van Vliet AK, van Gulik TM, Bennink RJ. ${ }^{99 \mathrm{~m}} \mathrm{Tc}-\mathrm{GSA}$ scintigraphy with SPECT for assessment of hepatic function and functional volume during liver regeneration in a rat model of partial hepatectomy. J Nucl Med. 2008; 49:122-128.

20. Kwon AH, Matsui Y, Kaibori M, Ha-Kawa SK. Preoperative regional maximal removal rate of technetium-99m-galactosyl human serum albumin (GSA-Rmax) is useful for judging the safety of hepatic resection. Surgery. 2006; 140:379-386.

21. Miki K, Kubota K, Inoue Y, Vera DR, Makuuchi M. Receptor measurements via Tc-GSA kinetic modeling are proportional to functional hepatocellular mass. J Nucl Med. 2001; 42:733-737.

22. Mitsumori A, Nagaya I, Kimoto S, Akaki S, Togami I, Takeda Y, Joja I, Hiraki Y. Preoperative evaluation of hepatic functional reserve following hepatectomy by technetium-99m galactosyl human serum albumin liver scintigraphy and computed tomography. Eur J Nucl Med. 1998; 25:1377-1382.

23. Kwon A, Ha-Kawa SK, Uetsuji S, Inoue T, Matsui Y, Kamiyama Y. Preoperative determination of the surgical procedure for hepatectomy using technetium-99mgalactosyl human serum albumin $\left({ }^{99 \mathrm{~m}} \mathrm{Tc}-\mathrm{GSA}\right)$ liver scintigraphy. Hepatology. 1997; 25:426-429.

24. Ha-Kawa SK, Tanaka Y, Hasebe S, Kuniyasu Y, Koizumi $\mathrm{K}$, Ishii Y, Yamamoto K, Kashiwagi T, Ito A, Kudo M, Ikekubo K, Tsuda T, Murase K. Compartmental analysis of asialoglycoprotein receptor scintigraphy for quantitative measurement of liver function: A multicentre study. Eur J Nucl Med. 1997; 24:130-137.

25. Kwon AH, Ha-Kawa SK, Uetsuji S, Kamiyama Y, Tanaka Y. Use of technetium $99 \mathrm{~m}$ diethylenetriaminepentaacetic acid-galactosyl-human serum albumin liver scintigraphy in the evaluation of preoperative and postoperative hepatic functional reserve for hepatectomy. Surgery. 1995; 117:429-434.

26. Miki K, Matsui Y, Teruya M, Kaminishi M, Kokudo N. Index of convexity: A novel liver function index using Tc-GSA scintigraphy. World J Gastroenterol. 2013; 19:92-96.

27. Morris-Stiff G, Tan YM, Vauthey JN. Hepatic complications following preoperative chemotherapy with oxaliplatin or irinotecan for hepatic colorectal metastases. Eur J Surg Oncol. 2008; 34:609-614.

28. Ryan P, Nanji S, Pollett A, Moore M, Moulton CA, Gallinger S, Guindi M. Chemotherapy-induced liver injury in metastatic colorectal cancer: Semiquantitative histologic analysis of 334 resected liver specimens shows that vascular injury but not steatohepatitis is associated with preoperative chemotherapy. Am J Surg Pathol. 2010; 34:784-791.

29. Zorzi D, Laurent A, Pawlik TM, Lauwers GY, Vauthey JN, Abdalla EK. Chemotherapy-associated hepatotoxicity and surgery for colorectal liver metastases. Br J Surg. 2007; 94:274-286.

(Received January 19, 2017; Revised march 17, 2017; Accepted April 2, 2017) 